\title{
Trisomy 21 Mosaicism
}

National Cancer Institute

\section{Source}

National Cancer Institute. Trisomy 21 Mosaicism. NCI Thesaurus. Code C142099.

The presence of cells with and without three copies of chromosome 21 in either somatic or germinal tissue. 